\title{
THE CONFORMAL MAPPING OF SIMPLY- CONNECTED RIEMANN SURFACES II ${ }^{*}$
}

\author{
MAURICE HEINS
}

1. On reviewing recently the proof which I gave for the Riemann mapping theorem for simply-connected Riemann surfaces several years ago [2], I observed that the argument which I used could be so modified that the assumption of a countable base could be completely eliminated. The problem of treating the Riemann mapping theorem without this assumption has been current for some time.** The object of the present note is to give an account of a solution of this question. Of course, the classical theorem of Rado permits us to dispense with an attack on the Riemann mapping theorem which does not appeal to the countable base assumption. In this connection, we recall that Nevanlinna [4] has given a straightforward potential-theoretic treatment of the Rado theorem in which neither the Riemann mapping theorem (nor the notion of a universal covering) enters as they do in Radó's proof. Nevertheless, a certain technical interest attaches to a direct treatment of the Riemann mapping theorem without the countable base assumption. An immediate byproduct of such a treatment is a simple proof of the Radó theorem which invokes the notion of a universal covering but in a manner different from that of Radó's proof. Indeed, it suffices to note that a manifold has a countable base if the domain of a universal covering does.

An essential role will be played in the exposition which follows by the classification of Riemann surfaces introduced by Ahlfors [1] according to which a Riemann surface is termed hyperbolic provided that there exists a nonconstant negative subharmonic function on the surface and is termed parabolic if this is not the case.

We put aside the situation of a compact surface since we have no reason to modify the exposition already given for this case. However we note that

Received May 14, 1957.

* This research was supported by the United States Air Force through the Office of Scientific Research of the Air Research and Development Command.

** (Added October 5, 1957). An entirely different treatment of this question is to be found in the monograph of G. Springer, "Introduction to Riemann Surfaces" which appeared recently. 
the potential-theoretic developments of [3] persist in the case of a non-compact parabolic Riemann surface. As a consequence of this fact, the difficulties inherent in freeing our earlier treatment of the parabolic case of the countable base assumption are circumvented.

2. Hyperbolic case. Although it is not explicitly stated, the considerations of [1] furnish a proof for the existence of a Green's function for a hyperbolic Riemann surface without the intervention of the countable base assumption. The treatment for the hyperbolic case of the mapping theorem given in [3] is applicable with the Green's function as defined by Ahlfors replacing the Green's function defined with the aid of exhaustions. One need only note that, if $g$ is the Green's function (in the sense of Ahlfors) for a hyperbolic surface $F$ with pole at $q \in F$ and $u$ is a positive superharmonic function on $F$ which satisfies $\lim \inf _{q}(u-g)>-\infty$, then $u \geqslant g$.

To be precise, we recall that the Green's function (in the sense of Ahlfors) for $F$ with pole at $q$ is the upper envelope of the class of functions $v$ with domain $F$ which satisfy: (1) $v$ is subharmonic in $F-\{q\},(2)$ if $\phi$ is a uniformizer satisfying $\phi(0)=q, v(\phi(z))+\log |z|$ is the restriction of a function subharmonic at $z=0$, (3) for each $\varepsilon>0,\{v \geq \varepsilon\}$ is relatively compact. For each $v$ and each pair of positive numbers $\varepsilon, \eta,(u-v)+\eta g>-\varepsilon$ on $F-\{q\}$. Hence $u \geq v$ and consequently $u \geqslant g$.

The remarks of this section are probably known and are included only for the sake of completeness.

3. Parabolic case. Let $F$ now denote a non-compact parabolic simplyconnected Riemann surface. Our first task is to treat the potential-theoretic preliminaries. In this connection, the fact that $F$ is simply-connected plays no role.

Let $\phi$ denote a uniformizer with domain $\{|z|<2\}$ and let $\Omega=F-\phi\{|z| \leqq 1\}$. There exists a unique bounded continuous function $u$ in $\bar{\Omega}$ which is harmonic in $\Omega$ and whose restriction to $\operatorname{fr} \Omega$ is an assigned continuous function $U$ on $\operatorname{fr} \Omega$. Further $u$ attains its maximum and minimum on fr $\Omega$ (cf. [4] p. 210, 6.10).

Unicity follows from the fact that, if $u$ vanishes on fr $\Omega$, the function with domain $F$ which is equal to $|u|$ on $\bar{\Omega}$ and to zero elsewhere is bounded and subharmonic and hence is identically zero since $F$ is parabolic.

For the existence of $u$ and the verification of the maximum and minimum 
principles for $u$, we observe that there exist continuous functions $u$, $u$ in $?$ which are respectively subharmonic and superharmonic in $\Omega$ and agree with $U$ on $\operatorname{fr} \Omega$ and, in addition, satisfy: $\min U=\min v \leqq v \leqq w \leqq \max w=\max U$. The existence of such functions follows from the possibility of solving the Dirichlet problem for an annulus. In fact, it suffices to define $v$ on $\phi\{1 \leqq|z| \leqq 3 / 2\}$ as the solution of the Dirichlet problem with boundary values $U$ on $\phi\{|z|=1\}$ and $\min U$ on $\phi\{\mid z=3 / 2\}$ and elsewhere on $\Omega$ as $\min U ; w$ is analogously constructed with $\max U$ replacing $\min U$. The desired function $u$ is simply the upper envelope of the continuous functions in $\Omega$ which agree with $U$ on fr $\Omega$ are subharmonic in $\Omega$ and are dominated by $w$.

It is now easily seen that the maximum principle holds for subharmonic functions in $\Omega$ which are bounded above in the following form:

Let $v$ be a subharmonic function in $Q$ which is bounded above and let $\bar{v}$ denote the upper limit function of $v$. Then $\sup v=\max _{\mathrm{fr} \Omega} \bar{v}$.

In fact, we put aside the trivial case, $v \equiv-\infty$, and have $-\infty<\max _{1+\Omega 2}$, $\leqq \sup v<+\infty$. Let $w$ now denote the function with domain $Q$ which is equal to the solution of the Dirichlet problem in $\phi\{1<|z|<3 / 2\}$ with boundary values $\max _{\mathrm{fr} \Omega} \bar{v}$ on $\phi\{|z|=1\}$ and $\sup v$ on $\phi\{|z|=3 / 2\}$ and elsewhere in $Q$ is equal to $\sup v$. Clearly $w$ is a superharmonic majorant of $v$. Now the upper envelope of the family of subharmonic functions in $Q$ which are dominated by $w$ is the constant function with value $\max _{\mathrm{fr \Omega}} \bar{v}$. The assertion follows.

We observe that the argument of [3] remains valid for non-compact parabolic surfaces when we now (using the notation of [3]) let $U_{p}$ denote the bounded solution of the Dirichlet problem for $F-\phi\{|z| \leqq \rho\}$ satisfying $U_{\rho}\left[\phi\left(\rho e^{2 \theta}\right)\right]=A(\rho) \cos \theta+B(\rho)$ where $A(\rho)$ and $B(\rho)$ are so chosen that the maximum and minimum of $U_{\rho}$ on $\phi\{|z|=1\}$ are respectively 1 and -1 . It is to be observed that there exists a decreasing sequence $\left\{\rho_{n}\right\}$ with $\lim \rho_{n}=0$ such that $\left\{U_{o_{n}}\right\}$ converges uniformly in $\phi\{0<|z|<2\}$ (i.e. uniformly on each compact subset). As a consequence of the maximum principle enunciated in the second paragraph of the present section, we are assured that $\left\{U_{p_{n}}\right\}$ converges uniformly on $\Omega$ and hence that $\left\{U_{\rho_{n}}\right\}$ converges uniformly in $F-\{\phi(0)\}$. The remainder of the argument given in [3] persists for the present situation. We conclude: there exists a harmonic function $U$ in $F-\{\phi(0)\}$ which is bounded in the complement of a compact neighborhood of $p(0)$ and which is 
such that $U[\phi(z)]$ admits a representation of the form $\}\left[\alpha z^{-1}\right]+h(z)$, where $\alpha$ is a complex number $\neq 0$ and $h$ is harmonic in $\{\mid z:<2\}$.

Thanks to the fact that $F$ is simply-connected, we are led to the conclusion that there exists a meromorphic function in $F$ which has a pole of order one at an assigned point $q \in F$ but no other poles in $F$ and which has the property that its real part is bounded on the complement of a compact neighborhood of $q$.

It follows that for some $g \in F$ there exists a meromorphic function $f_{q}$ in $F$ which has a pole of order one at $q$ but no other poles in $F$ and which is bounded on the complement of a compact neighborhood of $q$. Clearly the set $E$ of $q$ for which such an $f_{q}$ exists is open. Further $E$ is closed. To see this, let $r \in \bar{E}$ and let $\phi$ denote a uniformizer with domain $\{|z|<2\}$ which satisfies $\phi(0)=r$. For each $q \in \phi\{|z|<1\} \cap E$ there exists an $f_{q}$ which satisfies: $\max _{|z|=1}\left|f_{q} \circ \phi\right|=2, \min _{|z|=1}\left|f_{q} \circ \phi\right|=1$. There exists a sequence of such $q$, say $q_{k}$, satisfying $\lim q_{k}=r$ and such that with $f_{q_{k}}$ so normalized, $\left\{f_{a_{k}} \circ \phi\right\}$ converges uniformly in $\{0<|z|<2\}$ to a limit function which has at most a simple pole at zero and no others in $\{|z|<2\}$. Thanks to the maximum principle for subharmonic functions bounded above in an $\Omega$, we infer that $\left\{f_{q_{k}}\right\}$ converges uniformly in $F-\{r\}$ to an $f_{r}$. Hence $E$ is closed. Consequently $E=F$. There exist admitted $f_{q}$ for each $q \in F$. Since $F$ is parabolic, we infer that for a given $q \in F$ the $f_{q}$ are linear in one another.

The proof of the mapping theorem for the present case may now be completed along the lines given in $\$ 4$ of [2]. (It will be noted that there is an unessential difference between the functions considered here-they may have a zero-and the reciprocals of those considered in $\$ 4$ of [2] which do not.) We fix $f_{q_{0}}$ and ask for the set $\leftleftarrows$ of $q$ for which each $f_{q}$ admits a representation of the form $\lambda \circ f_{q_{0}}$ where $\lambda$ is a linear fractional transformation. Clearly $q_{0} \in \mathbb{E}$. We note that, given $f_{q}$, for $r(\neq q)$ sufficiently near $q,\left[f_{q}-f_{q}(r)\right]^{-1}$ is an $f_{1}$. It follows that $(5$ is both open and closed. Hence $(\xi ;=F$.

Suppose that $f_{q_{0}}(p)=f_{q_{0}}(r)$. From $f_{r}=\lambda \circ f_{q_{0}}$ we conclude $f_{r}(p)=f_{r}(r)$ and hence $p=r$. That is, $f_{q_{0}}$ is univalent. Since $F$ is non-compact parabolic, $f_{q_{0}}$ omits precisely one point of the extended plane. Hence $F$ is conformally. equivalent to the finite plane. 


\section{BIBLIOGRAPHY}

[1] Ahlfors; Lars V. On the characterization of hyperbolic Riemann surfaces. Ann. Acad. Sci. Fenn., I, 125 (1952).

[2] Heins, Maurice. The conformal mapping of simply-connected Riemann surfaces. Ann. of Math., (2) 50, 686-690 (1949).

[3] Heins, Maurice. Remarks on the elliptic case of the mapping theorem for simplyconnected Riemann surfaces. Nagoya Mathematical Journal, 9, 17-20, (1955).

[4] Nevanlinna, Rolf. Uniformisierung (1953).

Brown University and

Institutc for Advanced Study 